\title{
Monitorização de complicações pós-operatórias no ambiente domiciliar
}

\author{
Monitoring of postoperative complications in the home environment
}

Como citar este artigo:

Sousa AFL, Hermann PRS, Fronteira I, Andrade D. Monitoring of postoperative complications in the home environment. Rev Rene. 2020;21:e43161. DOI: https://doi.org/10.15253/2175-6783.20202143161

(DÁlvaro Francisco Lopes de Sousa ${ }^{1,3}$
(D)Paula Regina de Souza Hermann ${ }^{2}$
(DInês Fronteira ${ }^{3}$
(D) Denise de Andrade ${ }^{1}$

${ }^{1}$ Universidade de São Paulo, Escola de Enfermagem de Ribeirão Preto.

Ribeirão Preto, SP, Brasil.

${ }^{2}$ Universidade de Brasília. Brasília, DF, Brasil.

${ }^{3}$ Universidade Nova de Lisboa, Instituto de Higiene e

Medicina Tropical.

Lisboa, Portugal.

\section{Autor correspondente:}

Álvaro Francisco Lopes de Sousa

Avenida dos Bandeirantes, 3900 - Campus Universitário -

Monte Alegre.

CEP: 14040-902. Ribeirão Preto, SP, Brasil.

E-mail: alvarosousa@usp.br

\begin{abstract}
RESUMO
Objetivo: propor uma diretriz de monitorização de complicações pós-operatórias de pacientes no ambiente domiciliar. Métodos: pesquisa desenvolvida por meio da técnica Delphi com 45 profissionais de saúde brasileiros. Utilizaram-se o Google Forms para coleta de dados e a escala Likert para fins de consenso. Os dados foram analisados utilizando técnicas qualitativas (análise de conteúdo) e quantitativas (análise de frequências e percentuais). Resultados: foram elencadas 16 complicações elegíveis para serem utilizadas no monitoramento de pacientes cirúrgicos no pós-alta, sinais e sintomas a serem observados, frequência e tempo de monitoramento. De acordo com os juízes, é necessário o monitoramento, ao menos uma vez por dia $(68,8 \%)$, do egresso cirúrgico. 0 tempo máximo de monitoramento apresentou variação de 48 horas até 30 dias, no caso de risco de infecção. Conclusão: a diretriz se mostra válida para ser usada na detecção de complicações em egressos cirúrgicos no domicílio e antever a necessidade de reinternação.

Descritores: Procedimentos Cirúrgicos Operatórios; Complicações Pós-Operatórias; Monitoramento Epidemiológico; Infecção; Assistência Domiciliar.
\end{abstract}

\section{ABSTRACT}

Objective: to propose a guideline for monitoring postoperative complications in patients in the home environment. Methods: research developed using the Delphi technique with 45 Brazilian health professionals. Google Forms were used for data collection and a Likert scale was used for consensus purposes. The data were analyzed using qualitative (content analysis) and quantitative (frequency and percentage analysis) techniques. Results: sixteen complications eligible to aid in the monitoring of surgical patients after discharge were listed, as well as signs and symptoms to be observed, and frequency and time of monitoring. According to the judges, surgical patients must be monitored at least once a day $(68.8 \%)$. The maximum monitoring time varied from 48 hours to 30 days in case of risk of infection. Conclusion: the guideline is valid to be used to detect complications in surgical patients in the home environment and to anticipate the need for readmission.

Descriptors: Surgical Procedures, Operative; Postoperative Complications; Epidemiological Monitoring; Infection; Home Nursing. 


\section{Introdução}

A necessidade de aumentar a rotatividade dos leitos e otimizar recursos e despesas decorrentes da internação hospitalar tem significativa influência na decisão de alta dos pacientes. Em decorrência disso, observa-se aumento da alta hospitalar precoce e consequente transferência de cuidados para o ambiente domiciliar. Essa alta pode ser viabilizada sem o devido planejamento, expondo o paciente a complicações e, consequentemente, a readmissões e/ou óbitos. No caso de pacientes cirúrgicos no pós-operatório, essa assertiva se torna cada vez mais frequente e problemática, principalmente pela elevada probabilidade de complicações no domicílio ${ }^{(1)}$.

0 pós-operatório configura-se como um período de alto risco de complicações e compreende três etapas específicas: pós-operatório imediato, mediato e tardio. A primeira etapa, pós-anestésica, dura até 24 horas pós-cirurgia. A segunda é intermediária e corresponde ao período entre a fase pós-anestésica e a alta hospitalar (entre as 24 horas e os sete dias). Por fim, a terceira é a fase de convalescença que compreende o contínuo desde a alta hospitalar atẻ a recuperação esperada $^{(2)}$.

Embora os riscos tendam a predominar nos primeiros dias de pós-operatório, não são exclusivos deste. As complicações no pós-operatório tardio podem ter repercussões sérias, principalmente se o paciente se encontra no ambiente domiciliar. Diante disso, é destacado que a gravidade das complicações evidencia a necessidade de monitorização do paciente pela equipe de saúde, sendo imprescindível prevenir seus desdobramentos ${ }^{(1-3)}$.

Estudiosos afirmam que existem complicações comuns que podem ocorrer a todo paciente cirúrgico no pós-operatório, dentre as quais: problemas relacionados às vias aéreas, hipoxemia e embolia pulmonar, distúrbios circulatórios, como hemorragia e choque, distúrbios urinários, infecção do sítio cirúrgico, deiscência e evisceração ${ }^{(2-3)}$. Essas complicações costumam variar em termos de frequência e gravidade, sendo essas diferenças relacionadas ao tipo de cirurgia, condições e eventos do pré e pós-operatório, bem como fatores intrínsecos ao paciente.

No entanto, a inexistência de um sistema padronizado de vigilância e das complicações pós-alta dificulta estabelecer um panorama real da situação ${ }^{(3-5)}$, em termos de prevalência, intervenções e desfecho. Além disso, não há uma recomendação padronizada por órgãos nacionais de vigilância acerca do processo de vigilância e notificação dessas complicações ${ }^{(4-5)}$. Assim, entende-se que ainda há um "gap" entre o paciente pós-cirúrgico no domicílio e a equipe de saúde. Essa lacuna se dá pela dificuldade em contatar o paciente, classificar o nível de gravidade e, dessa forma, sistematizar ações que possam oportunizar cuidados ${ }^{(5)}$.

Objetivou-se propor uma diretriz de monitorização de complicações pós-operatórias de pacientes no ambiente domiciliar.

\section{Métodos}

Trata-se de uma pesquisa desenvolvida por meio da técnica Delphi. Essa técnica permite aos pesquisadores controlar e manipular as condições em que estão interessados, de forma que, com base no conhecimento e experiência profissional, busca-se uma mudança no valor de uma variável independente (opinião dos especialistas) e observa-se o efeito dessa mudança em outra variável dependente (complicações) ${ }^{(6)}$. A escolha da técnica se deu pela ausência na literatura de recomendações padronizadas sobre o monitoramento de complicações no pós-operatório cirúrgico no domicílio ${ }^{(5)}$.

Inicialmente, realizou-se uma revisão da literatura internacional ${ }^{(5)}$ objetivando fornecer um referencial adequado sobre as complicações comuns no pós-operatório tardio de pacientes submetidos à cirurgia, sua ocorrência, tempo de manifestação e intensidade. Os resultados dessa revisão foram utilizados para embasar o conteúdo proposto pelos juízes.

Na etapa metodológica de construção da diretriz, utilizou-se a amostra intencional, em que, para 
busca dos especialistas, consultou-se a Plataforma Lattes, pertencente ao Conselho Nacional de Ciência e Tecnologia, utilizando como instrumento de busca os currículos vitae e as palavras-chaves "Cirurgias" AND "complicações" com filtro para "Atuação profissional", aplicando os seguintes critérios: ser profissional médico ou enfermeiro; ter ao menos um artigo ou resumo em evento nacional ou internacional publicado sobre o tema nos últimos cinco anos; ser docente de curso de pós-graduação lato sensu na área há mais de cinco anos; e participar de grupos/projetos de pesquisa que envolvam as temáticas de interesse. Excluíram-se pesquisadores que tinham cadastro na Plataforma $L a$ ttes, mas pertenciam a outros países. Enviaram-se 152 convites, dos quais 107 não retornaram. Dessa forma, 45 profissionais médicos e enfermeiros responderam à primeira versão do instrumento.

Realizaram-se três rodadas, compreendidas entre setembro de 2018 e janeiro de 2019, conforme fluxograma (Figura 1). Na primeira rodada, enviou-se aos juízes um e-mail contendo o hiperlink para um for- mulário, criado pelos próprios autores, hospedado no Google Forms. Uma versão preliminar do formulário foi validada (face-conteúdo) por três juízes, que não fizeram parte das etapas posteriores.

0 formulário online encontrava-se dividido em duas seções: uma de caracterização sociodemográfica com informações que contemplavam a formação e atuação profissional; e outra com questões estruturadas em que os profissionais deviam indicar no mínimo três complicações cirúrgicas comuns à sua área de especialidade que pudessem ocorrer ao egresso cirúrgico já no domicílio (pós-operatório tardio). Ao final dessa rodada, tivemos um total de 58 complicações indicadas, acompanhadas de tempo necessário para monitorar, deadline de monitoramento e sinais e sintomas a serem observados. Os pesquisadores fizeram o compilado dessas complicações, listaram, excluíram as repetidas, agruparam e analisaram o material baseado nos resultados prévios do estudo de revisão ${ }^{(5)} \mathrm{e}$ princípios da análise de conteúdo ${ }^{(7)}$.

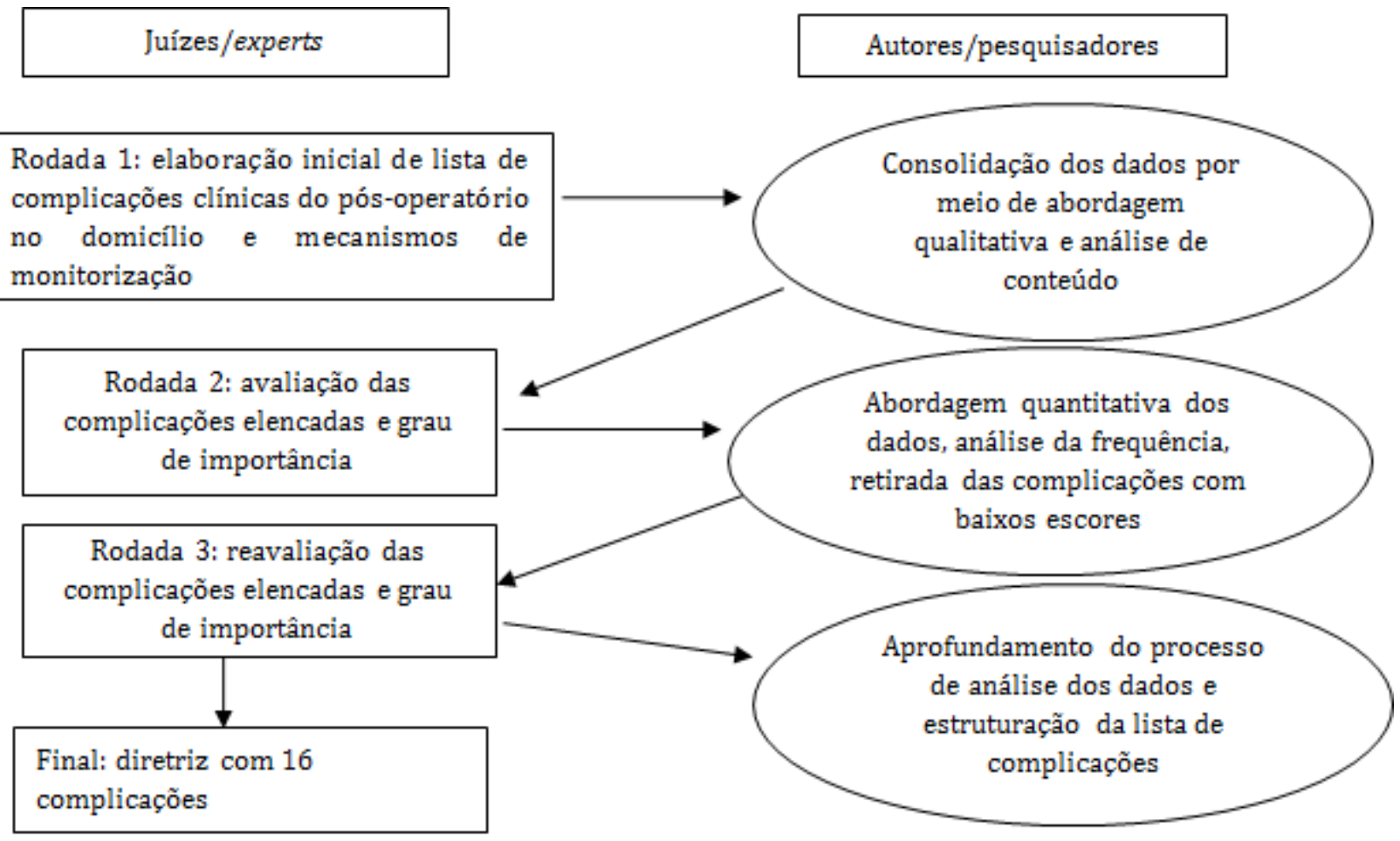

Figura 1 - Fluxograma de operacionalização da técnica Delphi. Ribeirão Preto, SP, Brasil, 2019 
Após análise sistemática, tivemos uma lista de 18 complicações que compuseram o formulário para análise pelos juízes na segunda rodada. Nessa rodada, já fornecemos as complicações sugeridas para que os juízes avaliassem o grau de importância atribuído a cada complicação por meio de uma escala tipo Likert (0-nenhuma, 1-muito pequena, 2-pequena, 3-razoável, 4-grande e 5-muito grande). Essa rodada teve adesão de 37 juízes, dos 45 iniciais. Ao final, analisamos as complicações de acordo com o índice de validade de conteúdo (IVC) e manteve-se uma complicação desde que a mesma tivesse percentual mínimo de 75,0\% de anotação nos escores "importante" ou "muito importante", de acordo com recomendações da literatura ${ }^{(8)}$, sendo excluídas duas complicações por não atingirem esse percentual.

Nesse contexto, na terceira rodada de avaliação de concordância, enviou-se aos juízes uma lista com as 16 complicações que atingiram o percentual mínimo de concordância. Excluíram-se complicações que não atingiram o grau de importância necessário na rodada anterior. Nessa rodada, participaram 34 juízes e todas as complicações tiveram escores acima de 80,0\%.

A cada rodada a lista de complicações era analisada de forma descritiva, segundo seu conteúdo, frequência e mediana. Na validação de conteúdo, analisou-se meticulosamente o conteúdo proposto, com o objetivo de verificar se os itens contemplados constituem uma amostra representativa do assunto que se deseja medir ${ }^{(9)}$.

0 projeto de pesquisa teve aprovação no comitê de ética da Escola de Enfermagem de Ribeirão Preto (Certificado de Apresentação para Aprecia ção Ética no 83275618.3.0000.5393 e protocolo no
2.756.298/2017), sendo a obtenção do Termo de Consentimento Livre e Esclarecido online.

\section{Resultados}

Neste estudo, participaram 12 médicos e 33 enfermeiros pertencentes a Instituições de Saúde e Ensino Superior das regiões Centro-Oeste $(n=3)$, Norte $(\mathrm{n}=2)$, Nordeste $(\mathrm{n}=13)$, Sul $(\mathrm{n}=9)$ e Sudeste $(\mathrm{n}=18)$. Os juízes escolhidos eram profissionais da área de ensino e assistência à saúde, com experiência e qualificação na área cirúrgica.

Dentre os juízes, $38(84,4 \%)$ eram do sexo feminino, 35 (77,8\%) possuíam doutorado, 28 (62,2\%) atuavam em Instituições de Ensino Superior, há menos de 20 anos, com foco nas seguintes especialidades: cirurgia geral, ortopédica, aparelho digestivo, cabeça e pescoço, torácica, cardiovascular, urológica e ginecológica.

O processo de apuração resultou em 16 complicações elegíveis para serem utilizadas no monitoramento de pacientes cirúrgicos no pós-alta hospitalar. A Figura 2 reúne o conjunto dessas complicações, frequência de monitoramento a ser realizado pela equipe de saúde, sinais e sintomas a serem observados/questionados e tempo de monitoramento.

De acordo com o consenso dos juízes, é necessário o monitoramento ao menos uma vez por dia, já que a maioria das complicações $(68,8 \%)$ exige monitoramento a cada 12 horas. Por outro lado, o tempo máximo de monitoramento apresentou maior variação, de 48 horas até 30 dias, no caso de infecção (Figura 2). 


\begin{tabular}{|c|c|c|c|}
\hline $\begin{array}{l}\text { Hipótese diagnóstica } \backslash \\
\text { Complicação }\end{array}$ & Sinais e possíveis sintomas & $\begin{array}{l}\text { Frequência de } \\
\text { monitoramen- } \\
\text { to (horas) }\end{array}$ & $\begin{array}{c}\text { Deadline (Fim } \\
\text { do monitora- } \\
\text { mento) }\end{array}$ \\
\hline Risco de infecção & $\begin{array}{l}\text { Febre elevada; presença de secreção purulenta na ferida operatória; dor } \\
\text { localizada; rubor e presença de abscessos }\end{array}$ & $12 / 12$ & 30 dias \\
\hline Sepse & Febre alta; hipotensão arterial e taquicardia & $12 / 12$ & 48 horas \\
\hline Hipertermia & Letargia; sonolência; sensação de frio e tremor (calafrio); sudorese e pele pálida & $12 / 12$ & 72 horas \\
\hline Dor & Relato verbal de dor & $12 / 12$ & 72 horas \\
\hline Desidratação & Vertigens; astenia; fadiga muscular; xerostomia; xeroftalmia e oligúria & $12 / 12$ & 72 horas \\
\hline Diurese reduzida (Oligúria) & $\begin{array}{l}\text { Rebaixamento do nível de consciência; alteração respiratória; distúrbio } \\
\text { metabólico por alteração hidroeletrolítica e edema }\end{array}$ & Diariamente & 72 horas \\
\hline Hipotermia & $\begin{array}{l}\text { Sensação de frio; tremores; espasmos musculares; pele fria e extremidades } \\
\text { cianótica }\end{array}$ & $12 / 12$ & 48 horas \\
\hline Atelectasia & $\begin{array}{l}\text { Frequência respiratória comprometida; baixa saturação de oxigênio e ausculta } \\
\text { pulmonar prejudicada }\end{array}$ & $12 / 12$ & 72 horas \\
\hline $\begin{array}{l}\text { Tromboembolismo pulmo- } \\
\text { nar }\end{array}$ & Dispneia; fadiga; saturação baixa e taquicardia & Diariamente & 5 dias \\
\hline $\begin{array}{l}\text { Insuficiência cardíaca con- } \\
\text { gestiva }\end{array}$ & $\begin{array}{l}\text { Dispneia (dor no peito; falta de ar ou tontura); sinal de infecção nas incisões } \\
\text { (saída de pus) }\end{array}$ & $12 / 12$ & 5 dias \\
\hline $\begin{array}{l}\text { Isquemia (membros inferio- } \\
\text { res) }\end{array}$ & $\begin{array}{l}\text { Dor; sensação de queimadura; cãibras musculares durante a marcha; palidez } \\
\text { cutânea do membro e perfusão diminuída }\end{array}$ & $12 / 12$ & 5 dias \\
\hline Trombose venosa profunda & $\begin{array}{l}\text { Vermelhidão ou descoloração na pele; dor local; edema; calor na perna e } \\
\text { distensão das veias da superfície do local afetado }\end{array}$ & $12 / 12$ & 7 dias \\
\hline Choque hipovolêmico & $\begin{array}{l}\text { Frequência respiratória comprometida; temperatura elevada; baixa saturação } \\
\text { de oxigênio; ausculta pulmonar prejudicada e mucosas ressecadas }\end{array}$ & Diariamente & 72 horas \\
\hline Arritmia cardíaca & Frequência cardíaca alterada; dor precordial e dispneia & Diariamente & 72 horas \\
\hline Deiscência & $\begin{array}{l}\text { Secreção serosa sanguinolenta (vermelho viva) ou purulenta através da ferida } \\
\text { operatória; rompimento de pontos }\end{array}$ & $12 / 12$ & 72 horas \\
\hline $\begin{array}{l}\text { Recuperação cirúrgica retar- } \\
\text { dada }\end{array}$ & $\begin{array}{l}\text { Náusea; vômito; sangramento; mobilidade física prejudicada; edema; problemas } \\
\text { com cateter ou material protético }\end{array}$ & Diariamente & 15 a 20 dias \\
\hline
\end{tabular}

Figura 2 - Principais complicações clínicas do paciente pós-cirúrgico no domicílio a serem investigadas segundo a natureza/tipo, frequência de monitoramento, deadline e sinais e sintomas observados. Ribeirão Preto, SP, Brasil, 2019

\section{Discussão}

Este estudo possui limitações relacionadas à dependência dos resultados à escolha correta dos juízes devido à possibilidade de introdução de viés de seleção. Também se destaca a possibilidade de forçar o consenso indevidamente, uma vez que o pesquisador é responsável por compilar os resultados a cada rodada antes de submeter novamente aos juízes. Para contornar tais limitações, reforçamos o critério de aleatorização na escolha dos juízes na plataforma Lattes. Além disso, a cada rodada os resultados eram confrontados com a literatura para evitar manipulações indevidas.

Em vários países, há um movimento de hospitalização domiciliar oportuna, principalmente do doente pós-cirúrgico. Assim, apesar das possíveis limitações, apresentar um consenso sobre quais complicações devem ser monitorizadas no contexto brasileiro e durante quanto tempo contribui com a prática clínica e 
pode ser usado no sentido de melhorar os desfechos e a qualidade dos cuidados prestados, bem como novas formas de prestar cuidados.

A intervenção cirúrgica nunca é isenta do risco de complicações em qualquer um dos seus mo$\operatorname{mentos}^{(3)}$, razão pela qual deve ser motivo de atenção da equipe de saúde. Estudos nacionais e internacionais ${ }^{(10-11)}$ mostram que a vigilância do paciente cirúrgico tem ocorrido prioritariamente durante o período em que ele permanece no hospital e mesmo quando amplia-se ao domicílio é feito de forma não sistematizada, geralmente com suporte telefônico e sem background científico válido de quais são as complicações mais graves e por quanto tempo devem ser monitoradas. Nesse sentido, esta pesquisa é pioneira ao propor uma série de complicações comuns a determinadas especialidades cirúrgicas e uma sistemática forma de avaliação da mesma.

Nossos achados reforçam essa assertiva, uma vez que as complicações aqui elencadas são apontadas em uma série de outros estudos de prevalência ${ }^{(3,11-12)}$. As principais complicações foram agrupadas em gerais, respiratórias, cardiocirculatórias, infecções, distúrbios cognitivos e outros, seguindo exemplo da literatura $^{(3,5,11)}$.

Dentre estas, as complicações pulmonares no pós-operatório se configuram na mais comum causa de morbidade pós-operatória, sobretudo em pessoas com mais de 60 anos, nas quais são a segunda causa de morte mais comum ${ }^{(3,13)}$. 0 destaque para as complicações pulmonares no pós-operatório ocorre pelo fato de estas serem comuns a uma série de especialidades cirúrgicas, tendo como principais representantes a pneumonia, atelectasia, insuficiência respiratória e tromboembolismo pulmonar, apontadas também em nosso estudo. As complicações que fazem parte desse grupo possuem incidência variando de 5 a $35,0 \%$ segundo dados recentes de estudos multicêntricos, com elevada mortalidade, em torno de $30,0 \%$, devido ao agravamento do quadro clínico com rapidez ${ }^{(13-14)}$.

Por outro lado, as complicações cardiocircula- tórias no pós-operatório possuem incidência menor que as primeiras e ocorrem em um pós-operatório mais precoce do que as complicações pulmonares graves, englobando situações como infarto agudo do miocárdio, insuficiência cardíaca congestiva, isquemia (membros inferiores) e trombose venosa profunda. Essas complicações tornam a recuperação mais prolongada e atrasam significativamente o retorno às atividades normais e, consequentemente, a diminuição da qualidade de vida ${ }^{(15-16)}$.

Após a cirurgia, os pacientes permanecem em jejum por certo período de tempo, mas com manutenção da hidratação parenteral pela anestesia cirúrgica e íleo adinâmico, mesmo nos casos que não envolvem o sistema digestivo. Por esse motivo, a presença de distúrbios hidroeletrolíticos é tão presente no pós-alta, sendo a desidratação um destaque na literatura ${ }^{(1,3,5)}$.

As infecções, aqui representadas pelas complicações "risco de infecção e sepse", de maneira geral, são um sério problema, pois podem aumentar a letalidade, a mortalidade, causar reintegração e mais custos hospitalares. Um bom seguimento do paciente no pós-alta é associado a menores taxas de complicações. No pós-operatório tardio, estudos de meta-análise e revisão ${ }^{(5,10)}$ apontam a infecção como complicação mais comum que exige, quase sempre, reintervenção ou cicatrização por segunda intenção. A presença de um quadro infeccioso também pode favorecer a ocorrência de outras complicações. Um exemplo disso é a deiscência, ruptura parcial ou mesmo total de todas as camadas da parede da incisão operatória ${ }^{(3,5,10,17)}$.

Os distúrbios de temperatura (hipertermia e hipotermia) são importantes e frequentes complicações em todo o pós-operatório. Alterações leves de temperatura podem resultar em desfechos desfavoráveis, sobretudo em cirurgias grandes, motivo pelo qual se configurem em um é um dos parâmetros fisiológicos que deve ser mais rigorosamente controlado. Segundo achados de um ensaio clínico chinês ${ }^{(18)}$, a variação da temperatura corporal durante o perioperatório afeta significativamente a função imunológica dos pacien- 
tes em um longo período pós-cirurgia, aumentando, assim, as chances de óbito.

A dor também foi elencada como uma importante complicação a ser monitorada no pós-alta. Trata-se de um parâmetro multidimensional e subjetivo de difícil mensuração, mas que é a principal causa de readmissão após cirurgias ambulatoriais. Nesse sentido, estudo com egressos de artroplastia de joelho e quadril ${ }^{(19)}$ apontou a dor como principal complicação no pós-operatório tardio (31,3\%). A avaliação da mesma no pós-operatório deve ser implementada com uso de escalas validadas e ser expandida ao domicílio.

Pelo exposto, fica claro que a transição do hospital para o domicílio pode ser um período de incerteza e de risco para muitos pacientes. Dessa forma, um correto follow-up torna-se uma ferramenta fundamental para assegurar que a continuidade de cuidados seja garantida com a mesma qualidade que seria em um ambiente hospitalar ${ }^{(4)}$. Nesse sentido, os enfermeiros são os profissionais que estão melhor posicionados para assegurar os cuidados fora das organizações hospitalares ${ }^{(5,17)}$.

Esse follow-up pode ser feito até mesmo por contato telefônico. Há evidências científicas de que contatar o paciente após a cirurgia proporciona tranquilidade, segurança, bem como a monitorização da recuperação, aliando a franca redução de custos e recursos, para as pessoas e suas famílias, mas também para as organizações prestadoras de cuidados de saú$\mathrm{de}^{(20)}$.

\section{Conclusão}

Elencamos um conjunto de complicações, com respectivos sinais e sintomas, frequência e deadline de monitoramento, comuns a egressos cirúrgicos no domicílio. É necessário monitoramento ao menos uma vez por dia, por no mínimo 30 dias. A diretriz se mostra válida para ser usada na detecção de complicações em egressos cirúrgicos no domicílio e antever a necessidade de reinternação.

\section{Agradecimentos}

A Coordenação de Aperfeiçoamento de Pessoal do Nível Superior, Processo: no 88881.186996/201801.

\section{Colaborações}

Sousa AFL, Hermann PRS, Fronteira I e Andrade D colaboraram na concepção do projeto de pesquisa, análise e interpretação dos dados, redação e revisão crítica relevante do conteúdo intelectual e na aprovação final da versão a ser publicada.

\section{Referências}

1. Mouchtouris N, Lang MJ, Barkley K, Barros G, Turpin J, Sweid A, et al. Predictors of hospitalassociated complications prolonging ICU stay in patients with low-grade aneurysmal subarachnoid hemorrhage. J Neurosurg. 2019; 3:1-7. doi: http:// dx.doi.org/10.3171/2019.1.JNS182394

2. Stracieri LDS. Cuidados e complicações pós-operatórias. Medicina [Internet]. 2008 [citado 2020 jan. 20]; 41(4):465-8. Disponível em: http://revista.fmrp.usp.br/2008/VOL41N4/SIMP_4Cuidados_e_complicacoes-posoperatorias.pdf

3. Koivisto JM, Saarinen I, Kaipia A, Puukka P, Kivinen K, Laine KM, et al. Patient education in relation to informational needs and postoperative complications in surgical patients. Int J Qual Health Care. 2019; pii:mzz032. doi: http://dx.doi. org/10.1093/intqhc/mzz032

4. Sousa AFL, Bim LL, Schneider G, Hermann PRS, Andrade D, Fronteira I. M-health in the surgical context: prospecting, review and analysis of mobile applications. Open Nurs J.2019;13:18-27. doi: http:// dx.doi.org/10.2174/1874434601913010018

5. Sousa AFL, Bim LL, Hermann PRS, Fronteira I, Andrade D. Complicações no pós-operatório tardio em pacientes cirúrgicos: revisão integrativa. Rev Bras Enferm. 2020; 72(3). [In Press].

6. Valle ARMC, Andrade D, Sousa AF, Carvalho PRM. Infection prevention and control in households: 
nursing challenges and implications. Acta Paul Enferm. 2016; 29(2):239-44. doi: http://dx.doi. org/10.1590/1982-0194201600033

7. Bardin L. Análise de conteúdo [Internet]. 2016 [citado 2020 jan. 20]. Disponível em: https:// madmunifacs.files.wordpress.com/2016/08/ anc3a1lise-de-contec3bado-laurence-bardin.pdf

8. Souza AC, Alexandre NMC, Guirardello EB. Psychometric properties in instruments evaluation of reliability and validity. Epidemiol Serv Saúde. 2017; 26(3):649-9. doi: http://dx.doi.org/10.5123/ s1679-49742017000300022

9. Leung K, Trevena L, Waters D. Content validation of the evidence-based nursing practice assessment tool. Nurse Res. 2018; 26(1):33-40. doi: http:// dx.doi.org/10.7748/nr.2018.e1544

10. Woelber E, Schrick EJ, Gessner BD, Evans HL. Proportion of surgical site infections occurring after hospital discharge: a systematic review. Surg Infect (Larchmt). 2016; 17(5):510-9. doi: http:// dx.doi.org/10.1089/sur.2015.241

11. Danwang C, Mazou TN, Tochie JN. Global prevalence and incidence of surgical site infections after appendectomy: a systematic review and meta-analysis protocol. BMJ Open. 2018; 8:e20101. doi: http://dx.doi.org/10.1136/bmjopen-2017-020101

12. Magill SS, O'Leary E, Janelle SJ, Thompson DL, Dumyati G, Nadle J, et al. Changes in prevalence of health care-associated infections in U.S. hospitals. N Engl J Med. 2018; 379(18):1732-44. doi: http:// dx.doi.org/10.1056/NEJMoa1801550

13. Sadeghi HA, Ghadrdoost B. Evaluation of pulmonary complication in patient with valvular heart surgery: clinical and laboratory significances. Eur Resp J. 2018; 52:PA4111; doi: http://dx.doi. org/10.1183/13993003.congress-2018.PA4111
14. Patel K, Hadian F, Ali A, Broadley G, Evans K, Horder $\mathrm{C}$, et al. Postoperative pulmonary complications following major elective abdominal surgery: a cohort study. Perioper Med Lond. 2016; 5:10. doi: http://dx.doi.org/10.1186/s13741-016-0037-0

15. Cohn SL, Ros NF. Comparison of 4 cardiac risk calculators in predicting postoperative cardiac complications after noncardiac operations. Am J Cardiol. 2018; 121(1):125-30. doi: http://dx.doi. org/10.1016/j.amjcard.2017.09.031

16. Golubovic M, Stanojevic D, Lazarevic M, Peric V, Kostic T, Djordjevic M, et al. A risk stratification model for cardiovascular complications during the 3-month period after major elective vascular surgery. Biomed Res Int. 2018; 2018:4381527. doi: http://dx.doi.org/10.1155/2018/4381527

17. Alecrim RX, Taminato M, Belasco A, Longo MCB, Kusahara DM, Fram D. Strategies for preventing ventilator-associated pneumonia: an integrative review. Rev Bras Enferm. 2019; 72(2):521-30. doi: http://dx.doi.org/10.1590/0034-7167-20180473

18. Shao L, Pang N, Yan P, Jia F, Sun Q, Ma W, et al. Control of body temperature and immune function in patients undergoing open surgery for gastric cancer. Bosn J Basic Med Sci. 2018; 18(3):289-96. doi: http://dx.doi.org/10.17305/bjbms.2018.2552

19. Sousa AFL, Oliveira LB, Carvalho HEF, Ribeiro IP, Fronteira I, Andrade D. Ocorrência de complicações no pós-operatório tardio de artroplastia de joelho e quadril. Rev Pesq Fundam Care Online. 2020. [In Press].

20. Kassmann BP, Docherty SL, Rice HE, Bailey Jr DE, Schweitzer M. Telephone follow-up for pediatric ambulatory surgery: parent and provider satisfaction. J Pediatr Nurs. 2016; 27(6):715-24. doi: https://doi.org/10.1016/j.pedn.2012.02.004 